\title{
Mapping morphological genes relative to molecular markers in lettuce (Lactuca sativa L.)
}

\author{
W. WAYCOTT* $\dagger$, S. B. FORT $\dagger \S$, E. J. RYDER $\dagger \&$ R. W. MICHELMORE $\ddagger$ \\ †USDA Agricultural Research Service, 1636 East Alisal, Salinas, CA 93915, U.S.A. and $\ddagger$ Department of Vegetable \\ Crops, University of California, Davis, CA 95616, U.S.A.
}

\begin{abstract}
Two $F_{2}$ populations were generated by crossing morphologically diverse genetic stocks in order to map 10 morphological traits relative to polymerase chain reaction-based molecular markers (RAPDs). Using one segregating population generated from crossing the experimental line, 'dwarf-2', with the butterhead cultivar, 'Saffier', the dwarf phenotype conditioned by the $d w f 2$ locus was mapped using bulked segregation analysis to within $38 \mathrm{cM}$ of the Adh3 locus. Using the second segregating population generated by crossing two experimental lines, $87-25-1 \mathrm{M} \times 87-109 \mathrm{M}$, nine traits [white seed $(w)$, brown seed $(b r)$, salmon flower colour $(s a)$, pale yellow flower colour $(p a)$, virescent juvenile leaf colour ( $v i)$, plump involucre $(p l)$, yellow seed $(y)$, one of two complementary genes for anthocyanin expression $(C$ or $G)$ and anthocyanin spotting $(R s)$ ] were linked to RAPD loci, but only six of them could be placed on an existing genetic map of lettuce generated by analysis of cv. 'Calmar' $\times \mathrm{cv}$. 'Kordaat'. A tenth trait, golden yellow $(g y)$, remained unlinked. Approximately a third of the RAPD markers analysed segregated in both the $87-25-1 \mathrm{M} \times 87-1090 \mathrm{M}$ and 'Calmar' $\times$ 'Kordaat' populations. In the genomic regions with multiple segregating loci in common, their relative orders and distances were mostly conserved. In one instance, linkage detected in the present study consolidated two separate groups on the earlier genetic map.
\end{abstract}

Keywords: bulked segregant analysis, gene mapping, lettuce, morphological traits, RAPD.

\section{Introduction}

Lettuce (Lactuca sativa L.) is a rosette plant which is harvested for its leaves. Genetic studies in this species have identified many morphological genes controlling leaf, flower and seed characteristics, as well as numerous genes for disease resistance (Robinson et al., 1983, Waycott \& Taiz, 1991, Michelmore et al., 1994, Ryder, 1996). Several of these characters have been shown to be linked (Ryder, 1975, 1983, 1989, 1992; Kesseli et al., 1994). However, few morphological genes have been placed on the genetic maps developed using molecular markers.

The majority of recent mapping studies on lettuce have focused on populations segregating for disease resistance (Landry et al., 1987, Paran \& Michelmore, 1993, Kesseli et al., 1994; Michelmore et al., 1994). These studies have produced genetic maps containing

*Correspondence and present address: Seminis Vegetable Seeds, 650 Leanna Dr, Arroyo Grande, CA 94320, U.S.A. E-mail: bill.waycott@svseeds.com

§Present address: Department of Vegetable Crops, University of California, Davis, CA 95616, U.S.A. isozyme, RFLP and RAPD markers as well as four major clusters of disease resistance genes. The core map was generated from an intraspecific cross between cultivars 'Calmar' and 'Kordaat' that is currently comprised of over 500 markers spanning more than $1200 \mathrm{cM}$, distributed in 13 major and four minor linkage groups (Kesseli et al., 1994 and unpubl. data). Additional populations have been analysed using bulked segregant analysis (BSA) (Michelmore et al., 1991) to map individual genes and develop linked markers (Kesseli et al., 1993).

In this paper, we report the mapping of 11 morphological genes of lettuce using two different mapping strategies and compare two intraspecific mapping populations for the similarity of their marker content and relative marker orders.

\section{Materials and methods}

\section{Generation of mapping populations}

One $F_{2}$ mapping population was constructed by crossing an $\mathrm{M}_{5}$ line of dwarf crisphead lettuce, 'dwarf-2' 
(Waycott \& Taiz, 1991), to a butterhead lettuce cultivar, 'Saffier'. 'Dwarf-2' is a GA-responsive dwarf mutant line $(d w f 2 d w f 2)$ that was generated by treating germinating seeds with ethyl methanesulphonate (Waycott et al., 1995). These parental lines were chosen on the basis of two criteria: (i) to facilitate mapping of the $d w f 2$ locus; and (ii) for similarity to the 'Calmar' $\times$ Kordaat' cross, which also produces a crisphead $\times$ butterhead mapping population. The latter consideration increased the probability that segregating marker loci would be shared between the 'dwarf-2' $\times$ 'Saffier' and 'Calmar' $x$ ' Kordaat' crosses, thereby allowing comparison and integration of linkage data. A total of $110 \mathrm{~F}_{2}$ plants from the 'dwarf-2' $\times$ 'Saffier' cross were grown in the greenhouse in 1986 and scored for the segregation of $d w f-2$. Subsequently, $110 \quad \mathrm{~F}_{3}$ lines were obtained by selfing each $\mathrm{F}_{2}$ plant, and 12 or more $\mathrm{F}_{3}$ individuals were grown to determine the genotypes of nondwarf $\mathrm{F}_{2} \mathrm{~S}$ as heterozygous or homozygous and for bulk harvest of leaf tissue for DNA. Leaf tissue was also collected from the parental lines and $F_{1}$. All samples were frozen at $-70^{\circ} \mathrm{C}$ prior to DNA isolation.

Lettuce lines $87-25-1 \mathrm{M}$ and $87-1090 \mathrm{M}$ were used as parents of the second mapping population. The pedigree of line $87-25-1 \mathrm{M}$ has contributions from crisphead, butterhead, leaf and Batavian lettuce types, whereas $87-1090 \mathrm{M}$ is of butterhead and primitive L. sativa parentage (Fig. 1). These parental lines were chosen based on allelic differences at 10 morphological loci. Line $87-25-1 \mathrm{M}$ is homozygous for alleles conditioning the traits white seed $(w)$, brown seed $(b r)$, (white seed is epistatic to brown seed), salmon flower colour ( $s a$ ), pale yellow flower colour $(p a)$, virescent juvenile leaf colour (vi) and plump involucre $(p l)$, whereas line $87-1090 \mathrm{M}$ is homozygous for alleles conditioning the traits yellow seed $(y)$, golden yellow leaf colour $(g y)$, one of two complementary genes for anthocyanin expression $(C$ or $G)$ and anthocyanin spotting $(R s)$. A total of $101 \mathrm{~F}_{2}$ plants from the $87-25-1 \mathrm{M} \times 87-1090 \mathrm{M}$ cross were grown in the greenhouse in 1992 and scored for the 10 morphological traits. $F_{3}$ families were also scored for these characters to determine the genotypes of the dominant $F_{2}$ phenotypes. Leaf tissues were collected from the parental lines and the $F_{1}$ and $F_{2}$ individuals and frozen at $-70^{\circ} \mathrm{C}$ prior to DNA isolation.

\section{DNA isolation and marker analysis}

DNA was extracted according to a modified CTAB method (Kesseli et al., 1994). Resuspended DNA samples were then diluted to a concentration of $15 \mu \mathrm{g} \mathrm{mL}^{-1}$ in sterile TE solution for use in PCR. The PCR reaction mixtures, DNA amplifications and electrophoresis gels were prepared according to previously established protocols for RAPDs (Waycott \& Fort, 1994). The 10-mer oligonucleotide primers used in PCR reactions for both mapping studies were a subset of sets A to $\mathrm{Y}$ from Operon Technologies (Alameda, CA). Linkage analyses and map construction were performed using MAPMAKER (Lander et al., 1987). A LOD threshold of 3.0 was used, unless otherwise indicated.

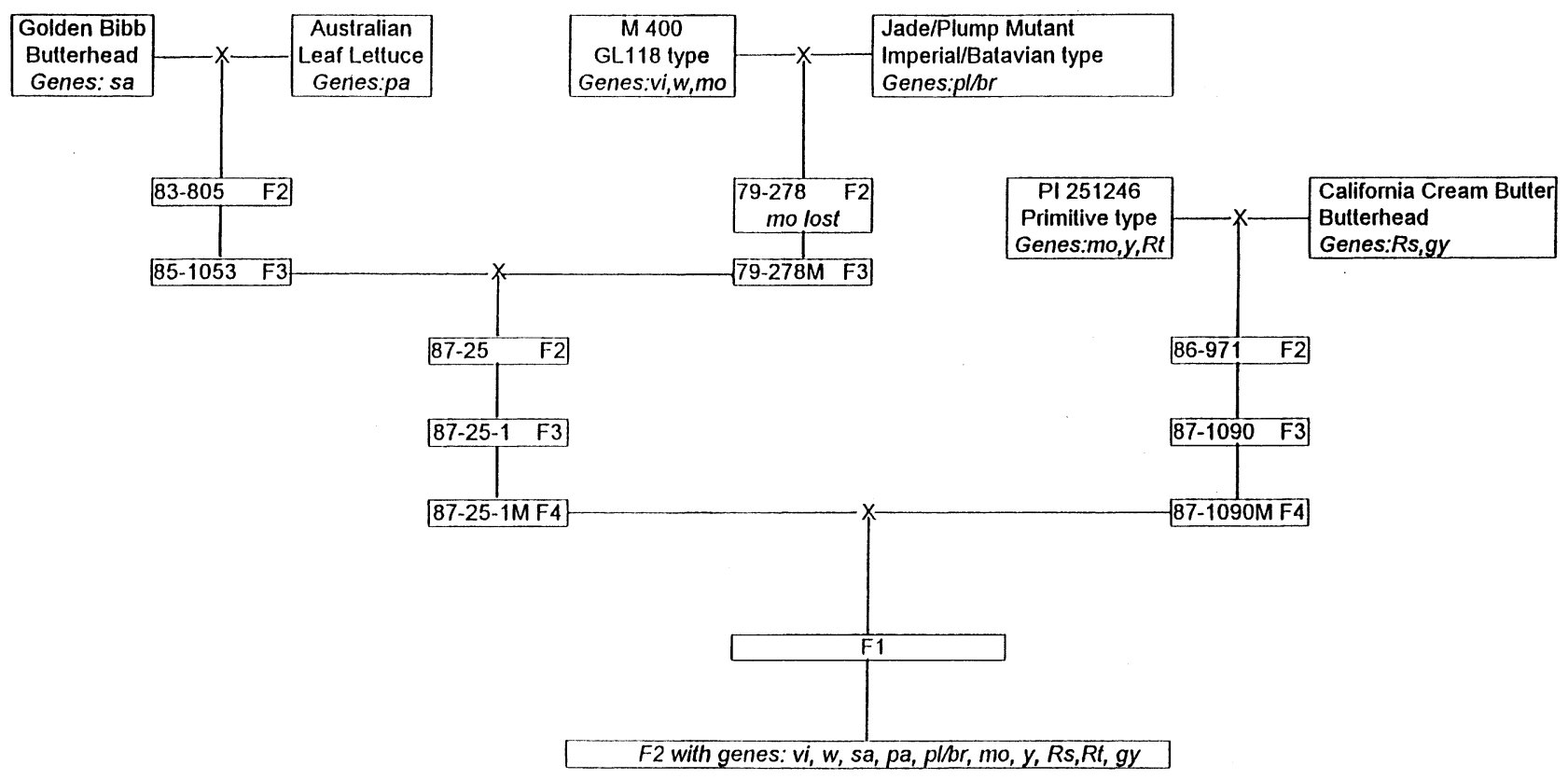

Fig. 1 Pedigree of $87-25-1 \mathrm{M} \times 87-1090 \mathrm{M}$ cross in Lactuca sativa L.

(C) The Genetical Society of Great Britain, Heredity, 82, 245-251. 


\section{Bulked segregant analysis of the 'dwarf 2' $\times$ 'Saffier' population}

For the 'dwarf-2' $\times$ 'Saffier' mapping study, contrasting DNA bulks of homozygous nondwarf ( $D w f 2 D w f 2)$ and dwarf ( $d w f 2 d w f 2)$ plants were formed by mixing equal amounts of diluted DNA from 8 to $12 \mathrm{~F}_{3}$ lines. These bulks were used as template DNA for identifying RAPD markers linked to the $d w f 2$ locus by bulk segregant analysis (BSA) (Michelmore et al., 1991). The segregation of RAPD markers that exhibited apparent polymorphisms between bulks was analysed using individual $\mathrm{F}_{3}$ lines and parental DNAs to determine linkage.

To place $d w f 2$ on the basic 'Calmar' $\times$ 'Kordaat' map and to identify additional linked markers, linked RAPD markers from 'dwarf-2' $\times$ 'Saffier' were analysed for polymorphism in the 'Calmar' $\times$ 'Kordaat' mapping population. Markers that showed differences between the 'Calmar' and 'Kordaat' parental lines were scored among the segregating $F_{2}$ plants and placed on the existing 'Calmar' $\times$ 'Kordaat' map. RAPD markers in the vicinity of the linked markers on the 'Calmar' $x$ 'Kordaat' map were analysed for polymorphism in the 'dwarf-2' $\times$ 'Saffier' population and then for linkage to the $d w f 2$ locus.

\section{Mapping studies in the $87-25-1 M \times 87-1090 M$ population}

As there were 10 characters segregating in the $F_{2}$ generation, the $87-25-1 \mathrm{M} \times 87-1090 \mathrm{M}$ population was analysed using an approach based on pre-existing linkage information rather than BSA. The RAPD markers used in construction of the linkage map were either: (i) RAPD markers known to be polymorphic in 'Calmar' $\times$ 'Kordaat'; or (ii) randomly selected primers that amplified multiple, bright-band polymorphisms between the $87-25-1 \mathrm{M}$ and $87-1090 \mathrm{M}$ parental lines. RAPD markers were scored by two independent observers prior to initiation of linkage analyses to ensure reliability of scoring. Linkage groups generated within $87-251 \mathrm{M} \times 87-1090 \mathrm{M}$ consisted of either dominant RAPD markers only or a mixture of dominant RAPD and codominant morphological markers. Map distances in linkage groups comprised exclusively of dominant RAPD markers were determined by linking sets of markers in coupling phase first, followed by joint analysis of markers in coupling and repulsion. Although rare $( \pm 5 \%)$, those RAPD markers in the joint analysis that drastically changed gene orders or map distances from the coupled analyses were removed from further consideration.

Markers that were polymorphic in both the 87-25$1 \mathrm{M} \times 87-1090 \mathrm{M}$ and 'Calmar' $\times$ 'Kordaat' populations served as a framework to place the 10 morphological loci mapped using $87-25-1 \mathrm{M} \times 87-1090 \mathrm{M}$ on the 'Calmar' $\times$ 'Kordaat' map. However, only those linkage groups constructed within the $87-25-1 \mathrm{M} \times 87-1090 \mathrm{M}$ mapping population that contained two or more RAPD markers from a single 'Calmar' $\times$ 'Kordaat' linkage group provided strong evidence that results from the two maps could be superimposed. Synteny based on only a single locus in common between the two maps was considered tentative because a polymorphic band from the same primer of similar size but different genetic position would result in an incorrect map position.

\section{Results}

\section{Bulked segregant analysis of the 'dwarf-2' $\times$ 'Saffier' population}

Over 200 primers were screened to map $d w f 2$ using pooled DNA samples from homozygous dwarf and nondwarf $\mathrm{F}_{3}$ families. Under our reaction conditions we detected an average of $6-10$ bands per primer; $\approx 30 \%$ were polymorphic between the parents. Therefore, over 400 polymorphic loci were sampled. Primer OPK02 identified a $710 \mathrm{bp}$ fragment that was polymorphic between the bulked DNA samples. OPK $022_{710}$ was also polymorphic in 'Calmar' $\times$ 'Kordaat' and was linked to two other RAPD loci, OPH $13_{860}$ and OPK $08_{1230}$, at distances of 13 and $6 \mathrm{cM}$, respectively, in the smaller un-numbered linkage group which contains Adh3 (Kesseli et al., 1994). Analysis of the 'dwarf 2' $\times$ 'Saffier' population with these additional markers showed OPK $08_{1230}$ to be the locus most closely linked to $d w f 2$ at a distance of $14.2 \mathrm{cM}$ (LOD 4.5); this placed $d w f 2$ approximately $38 \mathrm{cM}$ from Adh3 (Fig. 2). Neither OPH $13_{860}$ nor OPK $02_{710}$ was linked to $d w f 2$ at LOD scores in excess of 3.0, however, these markers were linked at LOD scores in excess of 2.0.

\section{Mapping of the 87-25-1M $\times 87-1090 \mathrm{M}$ population}

Seventy-six primers that identified evenly spaced polymorphisms in the 'Calmar' $x$ 'Kordaat' mapping population were screened for their ability to detect polymorphism in the $87-25-1 \mathrm{M} \times 87-1090 \mathrm{M}$ population. Of these 76 primers, 20 were informative. In addition, 56 arbitrary primers were surveyed for their ability to detect polymorphism; 13 of these were informative.

A total of 108 RAPD loci and 10 morphological markers were analysed. The segregation of 94 RAPD loci did not deviate significantly from a $3: 1$ ratio $(P>0.05)$. Of the 14 RAPD markers that exhibited significant segregation distortion at the $5 \%$ level, five showed distortion at the $1 \%$ level ( $\chi_{1}^{2}$ for 3:1 ratio). Of the morphological loci, none deviated significantly from 


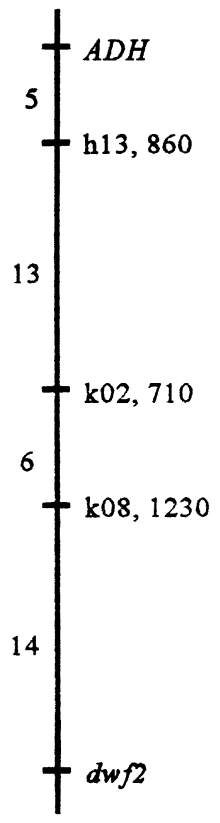

Fig. 2 RAPD loci and distances in cM between markers linked to $d w f 2$ in Lactuca sativa $\mathrm{L}$. Locus designations such as h13, $860=\mathrm{OPH} 13_{860}$ in text.

a 1:2:1 ratio. A total of 20 linkage groups of two or more linked RAPD markers were identified that spanned $987 \mathrm{cM}$. Nine of these groups contained six or more markers and comprised $579 \mathrm{cM}$. Only seven markers remained unlinked.

Linkages were detected between RAPD markers and all of the morphological markers except for golden yellow ( $g y)$ (Fig. 3) . Plump $(p l)$ and brown seed $(b r)$ were placed in the same linkage group and cosegregated; this result corresponds with a prior study (Ryder, 1975). Two RAPD markers within this linkage group $\left(\mathrm{OPN} 12_{920}\right.$, OPA10 870$)$ were shared with the 'Calmar' $\times$ 'Kordaat' mapping population, allowing these traits to be placed on linkage group 8 of the previous map (Kesseli et al., 1994). Virescent (vi) and white seed (w) were linked at $6 \mathrm{cM}$; this result also corresponds with a prior study ( Ryder, 1975). These traits were located to the same linkage group as three RAPD markers shared with the 'Calmar' $\times$ 'Kordaat' map $\left(\mathrm{OPC} 13_{2340}, \mathrm{OPD} 10_{1170}, \mathrm{OPJ} 04_{850}\right)$. Yellow seed $(y)$ was placed on the same linkage group as a single RAPD marker $\left(\mathrm{OPA} 01_{920}\right)$ putatively shared with the 'Calmar' $\times$ 'Kordaat' map on linkage group 2 . The other four traits, salmon flower colour $(s a)$, pale yellow flower colour $(p a)$, one of two complementary genes for anthocyanin expression ( $C$ or $G$ ), and anthocyanin spotting $(R s)$, were found to be linked to RAPD markers in the $87-25-1 \mathrm{M} \times 87-1090 \mathrm{M}$ population, but none of these markers were polymorphic in the 'Calmar' $\times$ 'Kordaat' cross.

A total of 24 RAPD markers were polymorphic in both the 'Calmar' $\times$ 'Kordaat' and 87-25-1M $\times$ 87$1090 \mathrm{M}$ populations, representing $20 \%$ of the markers analysed. The parental origins of these markers were analysed to see if any one genotype was preferentially amplified. Fourteen of the 24 shared markers were amplified from 'Kordaat'; of these, eight were amplified from $87-1090 \mathrm{M}$ and six from $87-25-1 \mathrm{M}$. The other 10 polymorphic markers were amplified from 'Calmar'; of these, four were amplified from $87-1090 \mathrm{M}$ and six from 87-25-1M. Therefore, polymorphic fragments were amplified from all genotypes at approximately equal frequency, and neither $87-25-1 \mathrm{M}$ nor $87-1090 \mathrm{M}$ appeared to resemble more closely 'Calmar' or 'Kordaat'. The distribution of positive alleles in 87-25-1M and 87$1090 \mathrm{M}$ was analysed to determine whether they contained segments that were similar to 'Calmar' or 'Kordaat'. There was no obvious clustering of positive alleles from either 'Calmar' or 'Kordaat'; therefore there was no evidence for recent identity by descent for any chromosome segment in these genotypes.

There were six cases in which two or more RAPD loci were linked in both the 'Calmar' $\times$ 'Kordaat' and 87-25$1 \mathrm{M} \times 87-1090 \mathrm{M}$ maps. In a total of 24 possible pairwise comparisons, the average linkage distances in the 87-25$1 \mathrm{M} \times 87-1090 \mathrm{M}$ and 'Calmar' $\times$ 'Kordaat' maps were 25.5 and $26.6 \mathrm{cM}$, respectively. The average difference between the 24 linkage distances for the two populations was $7.7 \mathrm{cM}$. In 12 out of the 24 comparisons, the difference was $4 \mathrm{cM}$ or less. Therefore, there was a fairly high correspondence of linkage distances across the two populations.

Conservation of locus orders could be assessed in the four linkage groups of $87-25-1 \mathrm{M} \times 87-1090 \mathrm{M}$ which contained three or more markers from the 'Calmar' $x$ 'Kordaat' map (Fig. 4). One shared linkage group, C08S03-L08-F12-H04-J04, differed only in the order of L8 and F12, and the total genetic distance differed by only $5 \mathrm{cM}$ ( 73 vs. $78 \mathrm{cM})$. In another linkage group, M05, $\mathrm{B} 08$ and $\mathrm{C} 11$ were linked in both maps, but their order was not conserved. Linkage of C13, D10 and J04 was similar in both maps; D10 and J04 cosegregated, with C13 closely linked. However, D10/J4 were proximate and $\mathrm{C} 13$ distal to white seed $(w)$ in 'Calmar' $\times$ 'Kordaat', whereas the reverse was observed for $87-25-1 \mathrm{M} \times 87$ 1090M. Lastly, R08-V14 and B10-B12 were mapped to separate linkage groups in 'Calmar' $\times$ 'Kordaat', whereas R08-V14-B10-B12 formed a single linkage group in $87-25-1 \mathrm{M} \times 87-1090 \mathrm{M}$. There were a few other instances where two loci on a linkage group were shared between maps, but not a third. 


\section{Discussion}

We were able to identify molecular markers linked to 10 out of the 11 morphological traits in the one-year period available to us. We were constrained to using RAPD markers because they are inexpensive and take less time to process than RFLPs, yet RAPDs proved efficient and effective for mapping these loci. Seven morphological loci were placed on the previous genetic map, contributing to the development of a detailed map for Lactuca sativa. Placement of the remaining loci will require analysis of additional markers.

The morphological markers were scattered throughout the map with no obvious clustering of leaf or reproductive traits, except for the cosegregation of the loci determining plump involucre $(p l)$ and brown seed $(b r)$. Reasons why these very different reproductive traits should be tightly linked is not apparent. The four leaf traits, virescent (vi), golden yellow $(g y)$, one of two complementary genes for anthocyanin expression $(C$ or $G)$, and anthocyanin spotting $(R s)$, were on separate linkage groups in this study. The golden yellow $(g y)$ trait was not allocated to any linkage group. Further work may show that it is on the same linkage group as one of the other genes.

Although the reproductive traits are of little horticultural significance, leaf colour is important in lettuce. The shade of green is important for most types of cultivated lettuce, and anthocyanin coloration is usually undesirable and is selected against. However, certain speciality types of lettuce have been developed which exhibit deep anthocyanin coloration and various pigmentation patterns. Markers linked to additional horticultural characteristics such as leaf shape and head (heart) formation as well as additional colour determinants would be useful. Because the expression of many of these commercially important genes cannot be scored during early stages of growth, the early and rapid determination of superior genotypes through markerassisted selection prior to field maturity would be of benefit to the breeder. As more markers are developed with linkages to horticultural and disease resistance

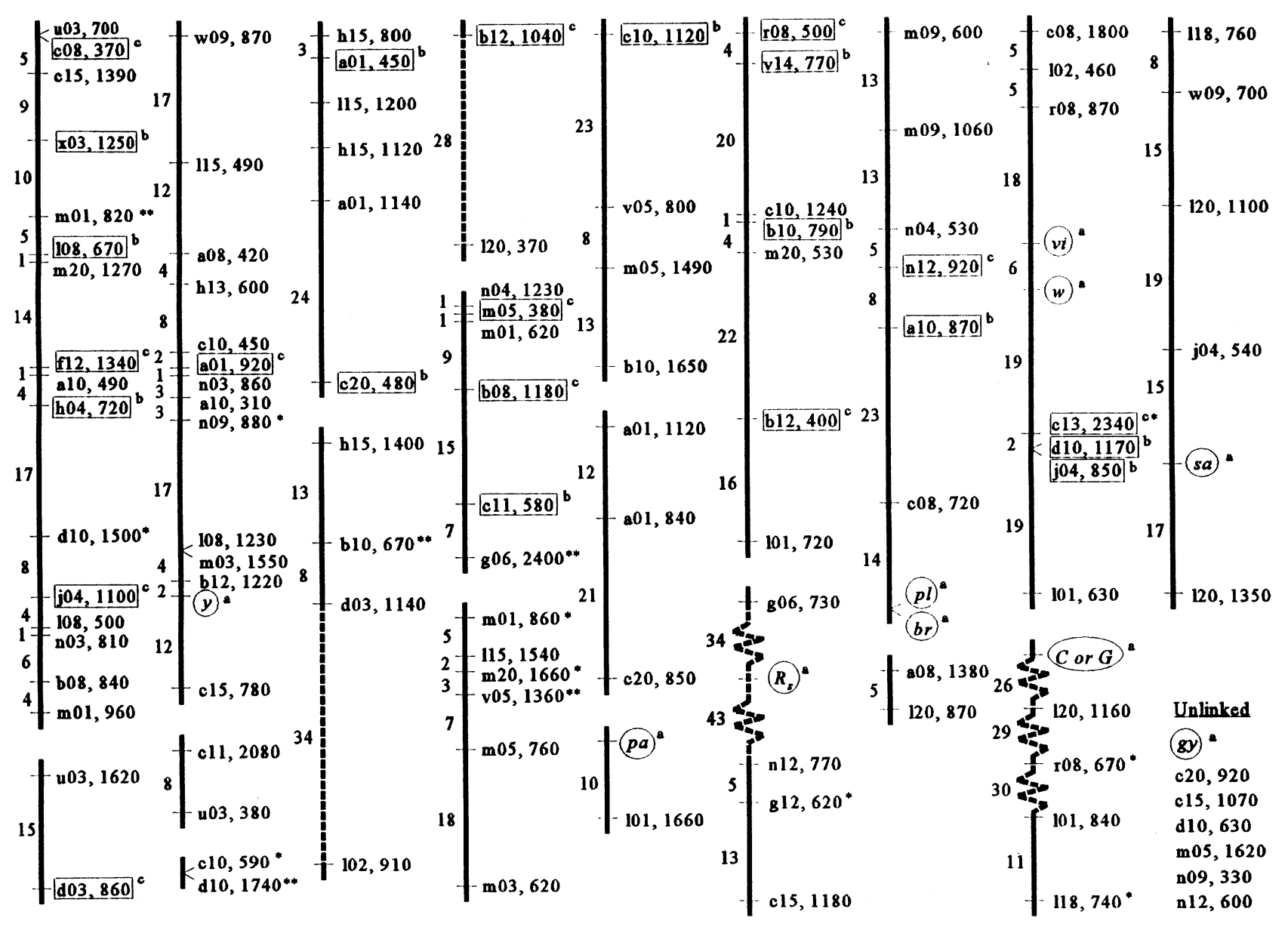

Fig. 3 Linkage groups and map locations for markers in the 87-25-1M $\times 87-1090 \mathrm{M}$ population of Lactuca sativa L. Dashed lines indicate linkages with LOD scores between 2.0 and 3.0.

(C) The Genetical Society of Great Britain, Heredity, 82, 245-251. 
traits, the cost/benefit ratio per assay becomes increasingly favourable.

Two different strategies were used for mapping morphological genes in this study. The first strategy, bulk segregant analysis, allowed us to identify the genetic position of $d w f 2$ but provided little additional information. The global linkage analysis used in the second cross allowed us to transfer linkage information from the pre-existing map to the $87-25-1 \mathrm{M} \times 87-1090 \mathrm{M}$ map via shared RAPD markers. These shared markers were used to generate a linkage backbone useful for mapping all segregating traits in the population simultaneously. We assumed that shared loci had the same genomic location in the two populations, but this could be verified only when two or more markers could be transferred from the same linkage group. The linkage groups were expanded by adding random loci identified by primers that had shown multiple bright, polymorphic bands in earlier studies. The second strategy was more efficient for mapping large numbers of genes than probing separate sets of bulked DNA for each trait. In addition, BSA would have greatly reduced our ability to compare maps derived from the two populations.

The percentage of RAPD markers in the 'Calmar' $\times$ 'Kordaat' map that were also polymorphic in $87-25-1 \mathrm{M} \times 87-1090 \mathrm{M}$ was low $(20 \%)$. The small proportion of markers segregating in both populations may have resulted primarily from the parentage of the

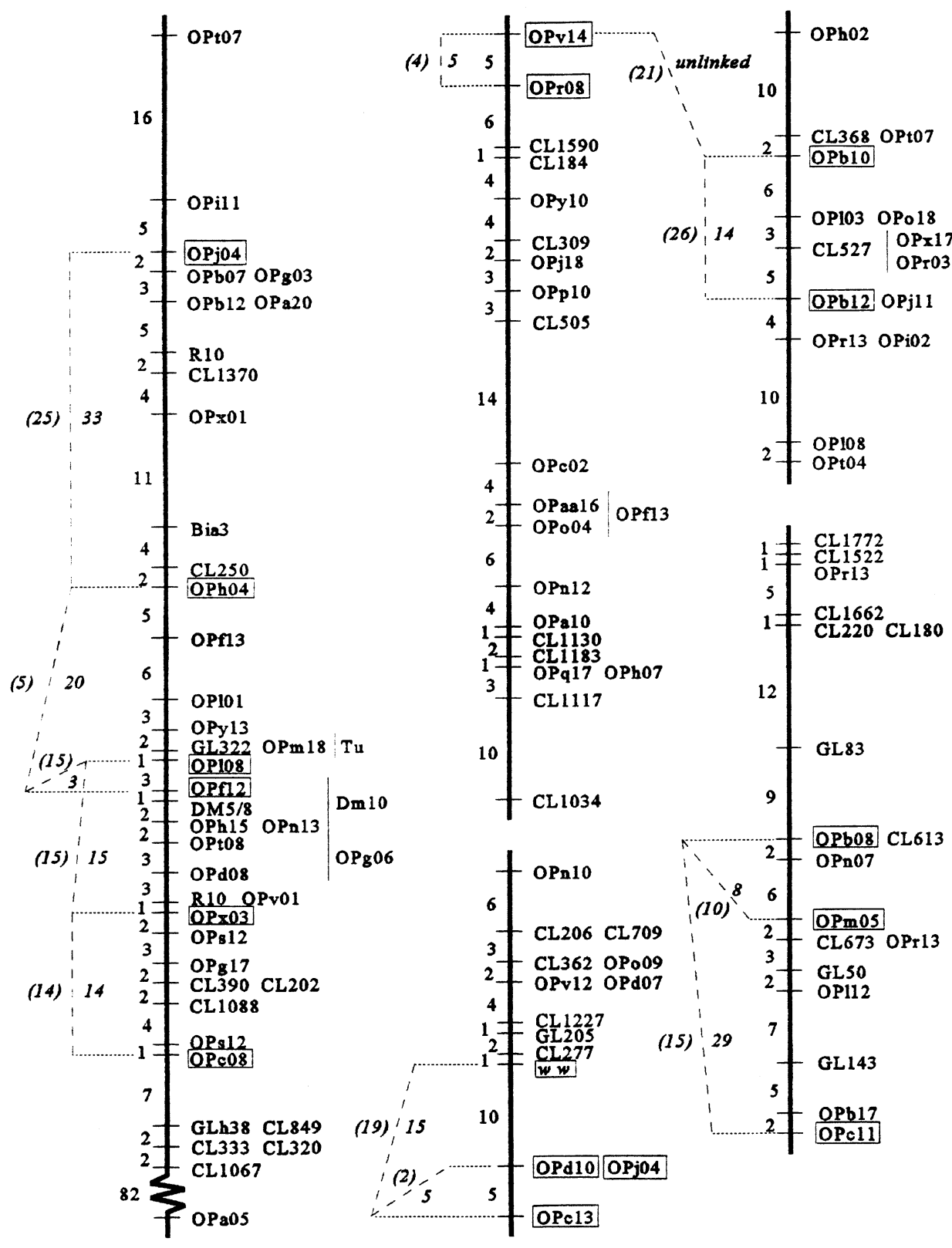

Fig. 4 Comparison of the 87-25$1 \mathrm{M} \times 87-1090 \mathrm{M}$ map and the 'Calmar' $\times$ 'Kordaat' map of Lactuca sativa $\mathrm{L}$. (excerpted above) in regions of conserved sequence homology. Markers polymorphic in both maps are indicated by boxes. Distances in cM for 87-25$1 \mathrm{M} \times 87-1090 \mathrm{M}$ (in parentheses) and 'Calmar' $\times$ 'Kordaat' are given to the left and right, respectively, of the dashed line.

(c) The Genetical Society of Great Britain, Heredity, 82, 245-251. 
$87-25-1 \mathrm{M} \times 87-1090 \mathrm{M}$ cross. The two parents in this cross had complex pedigrees, and both contained some proportion of butterhead origin (Fig. 1) . This partial commonality of genetic origins probably caused lower levels of polymorphism than if the two parents had been of completely different plant types. In addition, the cv. 'Australian' and PI 251246, ancestors of the 87$25-1 \mathrm{M} \times 87-1090 \mathrm{M}$ cross, are unrelated to either crisphead or butterhead genotypes (Fig. 1) . Their genetic contributions may have resulted in bands which were polymorphic in this cross, but not segregating in the 'Calmar' $x$ 'Kordaat' population.

Many of the markers used to construct the 'Calmar' $x$ 'Kordaat' map were RFLP loci, whereas all of the markers analysed in the $87-25-1 \mathrm{M} \times 87-1090 \mathrm{M}$ cross were RAPDs. Typically, RAPDs are dominant markers and RFLPs are codominant. Map intervals calculated between dominant markers vary greatly in precision according to whether linkages are in coupling or repulsion phase, whereas linkages between codominant markers are more accurate as recombinants are more readily detected. Despite these differences, we found that gene orders and linkage distances were fairly well maintained between the two maps. Of the major linked groups found in common, total linkage distances did not vary by more than $5 \mathrm{cM}$ and gene orders differed in only a few instances. Whether the apparent differences in gene order are the result of chromosomal rearrangements or artifacts arising from the statistical analysis of the mapping data would require characterization of additional populations.

\section{Acknowledgements}

We are grateful to Mr Salvador Placencia, Mr Bert Robinson, Mr Jacob Mann, Ms Luz García, Ms Margarita González-Chavera, Ms Jennifer Hillman, and Ms Keira Maiden for their assistance. We thank the California Iceberg Lettuce Advisory Board for their financial support.

\section{References}

KESSELI, R. V., WITSENBOER, H., VANDEMARK, G. J., STANGELLINI, M. E. AND MICHELMORE, R. W. 1993. Recessive resistance to Plasmopara lactucae-radicis maps by bulked segregant analysis to a cluster of dominant resistance genes in lettuce. Mol. Pl. Microbe Interact., 6, 722-728.

KESSELI, R. V., PARAN, I. AND MiChelmore, R. W. 1994. Analysis of a detailed genetic linkage map of Lactuca sativa (lettuce) constructed from RFLP and RAPD markers. Genetics., 136, 1435-1446.

LANDER, E. S., GREEN, P., ABRAHAMSON, J., BARLOW, A., DALY, M. J., LINCOLN, S. E. AND NEWBERG, L. 1987. Mapmaker: an interactive computer package for constructing primary genetic linkage maps of experimental and natural populations. Genomics, 1, 174-181.

LANDRY, B. S., KESSELI, R. V., FARRARA, B. AND MICHELMORE, R. W. 1987. A genetic map of lettuce (Lactuca sativa L.) with restriction fragment length polymorphism, isozyme, disease resistance and morphological markers. Genetics, 116, 331337.

MICHELMORE, R. W., PARAN, I. AND KESSELI, R. V. 1991. Identification of markers linked to disease resistance genes by bulked segregant analysis: a rapid method to detect markers in specific genomic regions using segregating populations. Proc. Natl. Acad. Sci. U.S.A., 88, 98289832.

MICHELMORE, R. W., KESSELI, R. V. AND RYDER, E. J. 1994. Genetic mapping in lettuce. In: Phillips, R. L. and Vasil, I. K. (eds) DNA Based Markers in Plants, pp. 223-239. Kluwer, Dordrecht.

PARAN, I. AND MICHELMORE, R. W. 1993. Identification of reliable PCR-based markers linked to disease resistance genes in lettuce. Theor. Appl. Genet., 85, 985-993.

ROBINSON, R. W., MCCREIGHT, J. D. AND RYDER, E. J. 1983. The genes of lettuce and closely related species. Pl. Breed. Rev., 1, 267-293.

RYDER, E. J. 1975. Linkage and inheritance in lettuce (Lactuca sativa L.). J. Am. Soc. Hort. Sci., 100, 346-349.

RYDER, E. J. 1983. Inheritance, linkage, and gene interaction studies in lettuce. J. Am. Soc. Hort. Sci., 108, 985-991.

RYDER, E. J. 1989. Studies of three new genes, linkage, and epistasis in lettuce. J. Am. Soc. Hort. Sci., 114, 129-133.

RYDER, E. J. 1992. Lettuce genetics: inheritance, linkage, and epistasis. J. Am. Soc. Hort. Sci., 117, 504-507.

RYDER, E. J. 1996. Inheritance of chlorophyll deficiency in lettuce. J. Hered., 87, 314-318.

WAYCOTT, W. AND FORT, s. B. 1994. Differentiation of nearly identical germplasm accessions by a combination of molecular and morphologic analyses. Genome, 37, 577-583.

WAYCOTT, w. AND TAIZ, L. 1991. Phenotypic characterization of lettuce dwarf mutants and their response to applied gibberellins. Pl. Physiol., 95, 1162-1168.

WAYCOTT, W., FORT, S. B. AND RYDER, J. R. 1995. Inheritance of dwarfing genes in Lactuca sativa L. J. Hered., 86, 39-44. 